\title{
Analysis of criteria for treatment initiation in patients with progressive chronic lymphocytic leukemia
}

\author{
Pablo Mozas', Alfredo Rivas-Delgado', Tycho Baumann', Neus Villamor ${ }^{2}$, Valentín Ortiz-Maldonado', Marta Aymerich², \\ Dolors Costa ${ }^{2}$, Alba Navarro², Eva Giné1,3, Armando López-Guillermo ${ }^{1,3}$, Emili Montserrat ${ }^{1}$ and Julio Delgado (1),3
}

\begin{abstract}
Chronic lymphocytic leukemia (CLL) is an indolent and incurable disorder characterized by the progressive accumulation of monoclonal B-cells in blood, bone marrow, and lymphoid organs. Since early intervention before the disease becomes symptomatic does not procure any benefit to patients ${ }^{1}$, treatment is only indicated in case of active disease, which is defined by the presence of marrow failure (MF), progressive lymphadenopathy, or splenomegaly (i.e., lymphoid mass (LM)), refractory immune cytopenia, general symptoms, or short lymphocyte doubling time $\mathrm{e}^{2-5}$. These criteria are largely based on clinical experience and have never been validated empirically. Moreover, some of these criteria correlate with clinicobiological features of the disease. For instance, lymphadenopathy is associated with 11q deletion and increased risk of Richter's transformation (RT) ${ }^{6}$. In addition, infiltrative cytopenia (as opposed to immune cytopenia) is associated with short survival ${ }^{7}$ and also with advanced age, which in turn predicts for a shorter survival ${ }^{8}$. The aims of this study were to evaluate the clinico-biological characteristics and outcome of patients with progressive CLL depending on the criterion that prompted first-line treatment.
\end{abstract}

After approval by the Institution's Review Board, we selected all consecutive patients from our institutional database requiring frontline therapy from 1978 to 2014, provided that they could be classified according to treatment criteria. These were: (i) progressive MF; (ii) massive

\footnotetext{
Correspondence: Julio Delgado (jdelgado@clinic.ub.es)

${ }^{1}$ Department of Hematology, Institute of Hematology and Oncology, Hospital

Clinic, Institut d'Investigacions Biomèdiques August Pi i Sunyer (IDIBAPS),

University of Barcelona, Barcelona, Spain

2Department of Pathology, Hematopathology Unit, Hospital Clínic, IDIBAPS,

University of Barcelona, Barcelona, Spain

Full list of author information is available at the end of the article
}

or progressive lymphadenopathy; (iii) massive or progressive splenomegaly; (iv) autoimmune anemia or thrombocytopenia unresponsive to standard therapy; (v) presence of general symptoms; and (vi) short lymphocyte doubling time ${ }^{2-5}$. Patients with progressive lymphadenopathy and those with progressive splenomegaly were difficult to separate from each other and were consequently grouped as "LM." Patients treated due to anemia or thrombocytopenia had their clinical records thoroughly investigated to correctly assign them to either MF or immune cytopenia. Fluorescent in situ hybridization (FISH) aberrations were classified following Döhner's hierarchical model and further grouped in low risk (normal, $13 \mathrm{q}-$ or +12$)$ and high risk $(11 \mathrm{q}-\text { or } 17 \mathrm{p}-)^{9}$. IGHV somatic mutations were evaluated following ERIC recommendations ${ }^{10}$, and NOTCH1, SF3B1, and TP53 mutations using previously described methods ${ }^{11}$.

Associations between baseline features were assessed by Fisher's exact test or Wilcoxon rank-sum test. Richter's transformation was calculated from frontline therapy using cumulative incidence curves, and the effect of each covariate was assessed using Gray's test. Overall survival (OS) was estimated from frontline therapy using Kaplan-Meier curves, and the effect of each covariate was assessed using the log-rank test. Complete-case multivariate analyses were modeled using the Cox regression equation, and the proportional hazards assumption was tested using Schönfeld residuals ${ }^{12}$. Apart from treatment criteria, the following covariates were evaluated: IGHV mutation status (mutated vs. unmutated); age (continuous); beta ${ }_{2}$-microglobulin (B2M, continuous), and FISH aberrations (low vs. high risk). Relative survival was computed using Spanish population data. All calculations were performed using R (version 3.2.4). Adjusted $P$ values 
$<0.05$ (Benjamini-Hochberg correction) were considered significant.

We identified 567 consecutive patients who received CLL-specific therapy, but information on treatment criteria was available in 530 (93\%) of them (Supplemental Table 1). Median age was 62 years (range, 22-93) at diagnosis and 65 years (range, 22-95) at frontline therapy, and $63 \%$ of patients were male. Treatment consisted of alkylating agents in $58 \%$, purine analogs in $21 \%$, purine analogs plus rituximab in $16 \%$, and other therapies in the remaining 5\%. Treatment eras were $1978-1990$ in $14 \%$, $1990-2000$ in $40 \%$, and $>2000$ in $46 \%$. Median follow-up from first-line therapy was 94 (range, 1-433) and 64 (range, 1-433) months for survivors and the entire population, respectively. Treatment criteria were MF in $31 \%$, LM in $72 \%$, refractory immune cytopenia in $3 \%$, general symptoms in $19 \%$, and short lymphocyte doubling time in 29\% (Supplemental Fig. 1). In total, 266/530 (50\%) patients had a single criterion, while the remaining 264 patients had two or more criteria for treatment initiation. Of note, 506/530 (95\%) patients were treated due to MF, LM, or both, with or without additional criteria, while only $24(5 \%)$ patients received therapy due to refractory immune cytopenia, general symptoms, short lymphocyte doubling time, or a combination of them (Supplemental Fig. 1). Consequently, we focused our analysis on the 506 patients treated due to MF, LM, or both. Patients who received therapy due to both MF and LM (47/506 (9\%)) were classified as MF following the same logic behind Binet and Rai staging systems ${ }^{13,14}$.

Compared to LM patients, MF patients were significantly older, had a significantly higher B2M concentration, and received alkylating agents more frequently (Supplemental Table 1). As expected, the MF population had a significantly lower hemoglobin concentration and platelet count at diagnosis, which was associated with a more advanced disease in terms of Rai or Binet clinical stages. There were a few cases of Binet stage $\mathrm{C}(n=7)$ and Rai stage III-IV $(n=8)$ disease in the LM population, which were due to immune cytopenia. The LM group showed a higher proportion of adverse prognostic features compared to the MF group, including unmutated $I G H V$ genes $(P=0.001)$, high ZAP70 expression $(P=0.001)$, and presence of $11 \mathrm{q}$ deletion $(P=0.05)$, although biomarkers were only available in around $60 \%$ of patients. In contrast, 13q deletions were more frequently detected in patients with MF $(P=0.03)$. Missing results were equally distributed across both groups $(P=0.15)$.

The median OS of the entire population was 77 months (95\% confidence interval (CI): 71-83) from first-line therapy and 108 months (95\% CI: 102-117) from diagnosis. When we evaluated each treatment criterion individually (i.e., patients who had that criterion vs. those who did not), we observed that both MF and LM had a significant impact on OS $(P<0.001$ for both comparisons, Fig. 1a, d). We also observed a trend toward a shorter survival for patients treated due to immune cytopenia (Fig. 1e, $P=0.06$ ). Of note, MF was associated with a shorter OS compared to the other treatment criteria pooled together, whereas LM had the opposite effect (i.e., it was associated with a longer OS compared to the other criteria pooled together). Moreover, since MF and LM were present in $95 \%$ of patients, and Fig. 1a looked like the specular reflection of Fig. 1d, we combined both criteria into one covariate called "treatment criteria" (LM vs. MF). In this subset comprising $95 \%$ of the original population, the median OS for MF patients was 63 months (95\% CI: 48-72) compared to 89 months (95\% CI: 80-106) for LM patients $(P<0.001$, Fig. 2a). Treatment criteria (MF vs. LM) remained a significant predictor of $O S$ after adjusting by age (70 years or less vs. $>70$ years; $P<0.001)$, B2M $(2.4$ $\mathrm{mg} / \mathrm{L}$ or less vs. $>2.4 \mathrm{mg} / \mathrm{L} ; P=0.002), I G H V$ mutational status (mutated vs. unmutated; $P<0.001$ ), FISH aberrations (low vs. high risk; $P=0.012$ ), or frontline therapy (alkylating agents vs. purine analogs; $P<0.001$ ). By multivariate analysis, three covariates had an independent impact on OS: age (hazard ratio (HR) 1.04, 95\% CI: $1.03-1.05, P<0.001$ ), B2M (HR 1.07, 95\% CI: $1.03-1.12, P$ $<0.001$ ), and treatment criteria ( $\mathrm{HR} 1.29,95 \% \mathrm{CI}$ : 1.01-1.66, $P=0.041)$. B2M violated the proportional hazards assumption $(P=0.026)$ and was removed from the model. When the multivariate analysis was repeated including B2M as a stratum, we confirmed that both age (HR 1.04, 95\% CI: 1.03-1.06, $P<0.001$ ) and treatment criteria (HR 1.38, 95\% CI: $1.02-1.88, P=0.038$ ) retained their statistical significance. Other covariates, such as IGHV mutation status or FISH aberrations were not included in the model because they were only available in around $60 \%$ of patients.

Since the studied population spanned many decades, we evaluated whether the impact of treatment criteria was independent of patient's age, sex, or calendar date by modeling the relative survival of our cohort. Both age and treatment criteria remained statistically significant $(P<$ 0.001 and $P=0.0233$, respectively). CLL-specific and normal population mortality plots confirmed that patients requiring therapy had a significantly higher mortality compared to the normal population, and that MF patients had a significantly higher CLL-specific mortality than LM patients (Fig. 2b).

Since bulky lymphadenopathy has been defined as a factor linked to the development of RT, we evaluated whether treatment criteria (LM vs. MF) was associated with that outcome. However, the cumulative incidence of transformation was not significantly affected by this covariate. As such, the incidence of transformation 10 years after treatment initiation was $12 \%$ (95\% CI 9-16\%) for the LM cohort compared to $7 \%$ (95\% CI $4-12 \%$ for the MF 

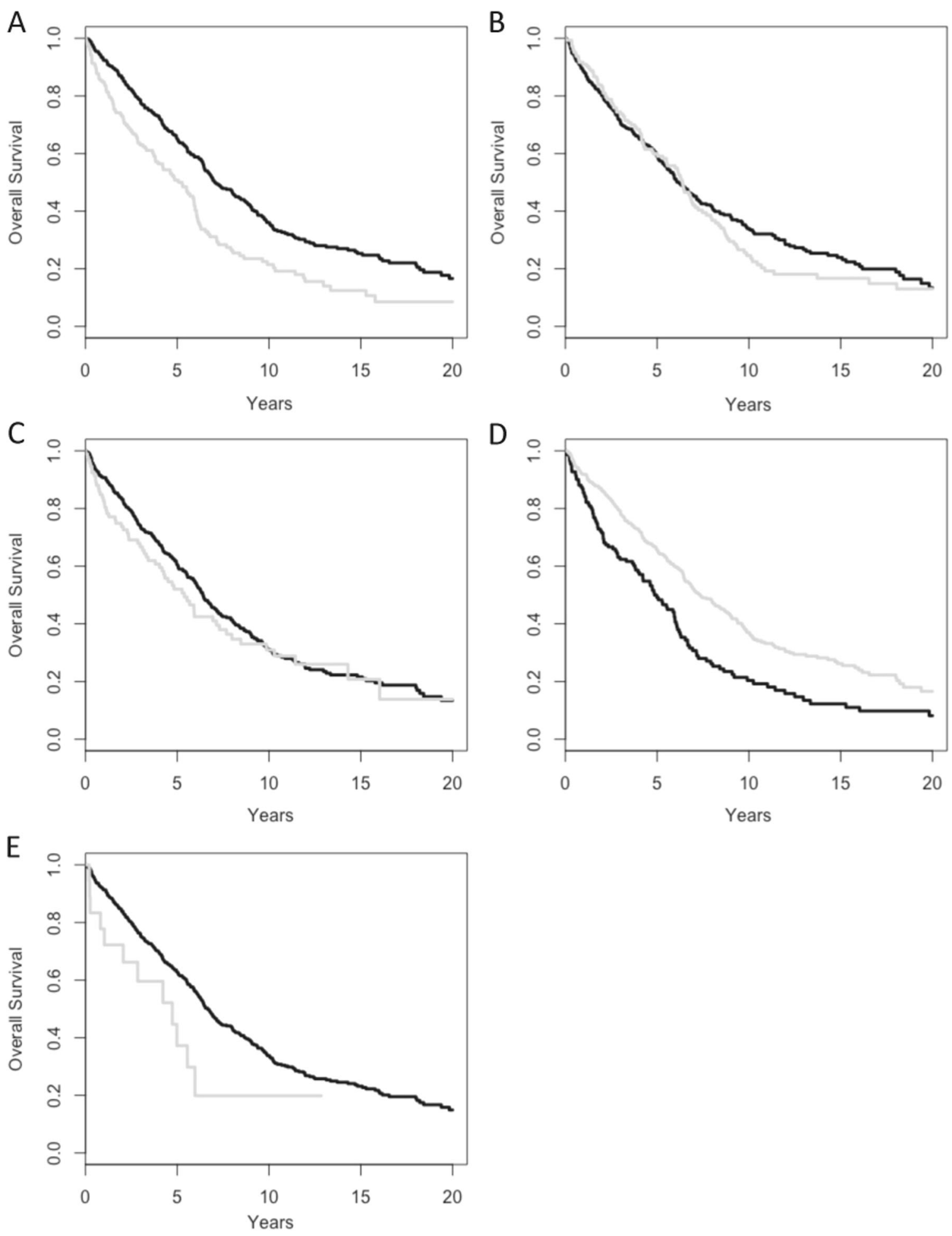

Fig. 1 Overall survival for the entire patient population according to each treatment criterion individually. a Marrow failure $(P<0.001)$; $\mathbf{b}$ short doubling time $(P=0.4)$; c general symptoms $(P=0.4)$; d lymphoid mass $(P<0.001)$; and $\mathbf{e}$ autoimmune hemolytic anemia $(P=0.06)$. In all plots, the gray curve represents the presence and the black curve the absence of that particular criterion. $P$ values were adjusted using the Benjamini-Hochberg method 

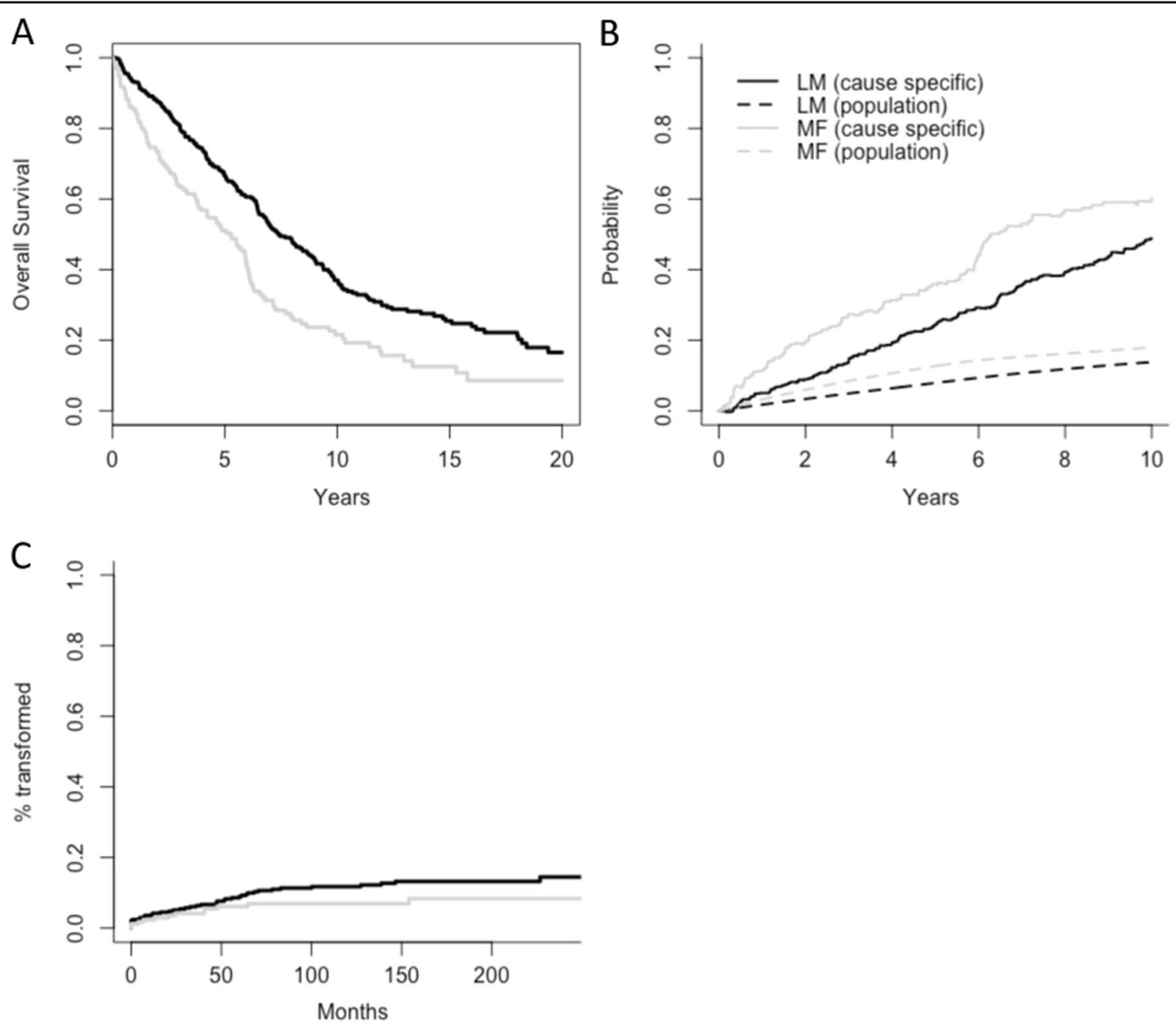

Fig. 2 Overall survival, CLL-specific mortality and incidence of Richter's transformation according to treatment criteria. a Overall survival according to treatment criteria, considering only patients treated because of marrow failure (MF), lymphoid mass (LM), or both ( $95 \%$ of the entire population). Patients who fulfilled both criteria were assigned to the MF group $(P<0.001)$. $\mathbf{b}$ CLL-specific mortality curves according to treatment criteria. Normal population mortality curves are also plotted for reference. Notice that the MF-reference mortality is slightly higher than the LMreference mortality because this population is significantly older. c Cumulative incidence of Richter's transformation according to treatment criteria $(P$ $=0.20$ ). MF patients are depicted in gray and LM patients are depicted in black

cohort $(P=0.20$, Fig. 2c)). There was borderline significance for high-risk FISH aberrations $(11 \mathrm{q}-$ or $17 \mathrm{p}-$, $P=0.09)$ and $I G H V$ mutational status $(P=0.12)$, and a clear statistical significance for NOTCH1 mutations $(P<$ 0.001, Supplemental Fig. 2), which is in keeping with previous reports ${ }^{15}$.

In summary, this study shows that patients with CLL treated because of LM have a better outcome than those in whom therapy is initiated due to MF. This retrospective analysis derives from a large patient population treated over a long period of time with standard chemotherapy or, more recently, chemo(immuno)therapy regimens available at each time period. Whether the observations presented here hold for novel therapies whose main therapeutic effect is observed in lymph nodes (i.e., B-cell receptor inhibitors) should be prospectively investigated.

\section{Author details}

'Department of Hematology, Institute of Hematology and Oncology, Hospital Clinic, Institut d'Investigacions Biomèdiques August Pi i Sunyer (IDIBAPS), University of Barcelona, Barcelona, Spain. 'Department of Pathology, Hematopathology Unit, Hospital Clínic, IDIBAPS, University of Barcelona, Barcelona, Spain. ${ }^{3}$ CIBERONC, Madrid, Spain

\section{Conflict of interest}

The authors declare that they have no conflict of interest.

\section{Publisher's note}

Springer Nature remains neutral with regard to jurisdictional claims in published maps and institutional affiliations. 
Supplementary Information accompanies this paper at https://doi.org/ 10.1038/s41408-017-0044-5.

Received: 14 September 2017 Revised: 16 November 2017 Accepted: 29 November 2017

Published online: 16 January 2018

\section{References}

1. Dighiero, G. et al. Chlorambucil in indolent chronic lymphocytic leukemia. French Cooperative Group on chronic lymphocytic leukemia. N. Engl. J. Med. 338, 1506-1514 (1998).

2. Silver, R. T., Sawitsky, A., Rai, K., Holland, J. F. \& Glidewell, O. Guidelines for protocol studies in chronic lymphocytic leukemia. Am. J. Hematol. 4, 343-358 (1978).

3. Cheson, B. D. et al. Guidelines for clinical protocols for chronic lymphocytic leukemia: recommendations of the National Cancer Institute-sponsored working group. Am. J. Hematol. 29, 152-163 (1988).

4. Cheson, B. D. et al. National Cancer Institute-sponsored Working Group guidelines for chronic lymphocytic leukemia: revised guidelines for diagnosis and treatment. Blood 87, 4990-4997 (1996).

5. Hallek, M. et al. Guidelines for the diagnosis and treatment of chronic lymphocytic leukemia: a report from the International Workshop on Chronic Lymphocytic Leukemia updating the National Cancer Institute-Working Group 1996 guidelines. Blood 111, 5446-5456 (2008).
6. Döhner, H. et al. 11q deletions identify a new subset of B-cell chronic lymphocytic leukemia characterized by extensive nodal involvement and inferior prognosis. Blood 89, 2516-2522 (1997).

7. Moreno, C. et al. Autoimmune cytopenia in chronic lymphocytic leukemia: prevalence, clinical associations, and prognostic significance. Blood 116, 4771-4776 (2010)

8. Baumann, T. et al. Chronic lymphocytic leukemia in the elderly: clinicobiological features, outcomes, and proposal of a prognostic model. Haematologica 96, 1599-1604 (2014).

9. Döhner, H. et al. Genomic aberrations and survival in chronic lymphocytic leukemia. N. Engl. J. Med. 343, 1910-1916 (2000).

10. Ghia, P. et al. ERIC recommendations on IGHV gene mutational status analysis in chronic lymphocytic leukemia. Leukemia 21, 1-3 (2007).

11. Santacruz, R. et al. The prognostic impact of minimal residual disease in patients with chronic lymphocytic leukemia requiring first-line therapy. Haematologica 99, 873-880 (2014).

12. Delgado, J., Pereira, A., Villamor, N., López-Guillermo, A. \& Rozman, C. Survival analysis in hematologic malignancies: recommendations for clinicians. Haematologica 99, 1410-1420 (2014).

13. Rai, K. R. et al. Clinical staging of chronic lymphocytic leukemia. Blood $\mathbf{4 6}$ 219-234 (1975).

14. Binet, J. L. et al. A new prognostic classification of chronic lymphocytic leukemia derived from a multivariate survival analysis. Cancer 48, 198-206 (1981).

15. Villamor, N. et al. NOTCH1 mutations identify a genetic subgroup of chronic lymphocytic leukemia patients with high risk of transformation and poor outcome. Leukemia 27, 1100-1106 (2013). 